\title{
Author Correction: Ultrasensitive detection of circulating tumour DNA via deep methylation sequencing aided by machine learning
}

Naixin Liang, Bingsi Li, Ziqi Jia, Chenyang Wang, Pancheng Wu, Tao Zheng, Yanyu Wang, Fujun Qiu, Yijun Wu, Jing Su, Jiayue Xu, Feng Xu, Huiling Chu, Shuai Fang, Xingyu Yang, Chengju Wu, Zhili Cao, Lei Cao, Zhongxing Bing, Hongsheng Liu, Li Li, Cheng Huang, Yingzhi Qin, Yushang Cui, Han Han-Zhang, Jianxing Xiang, Hao Liu, Xin Guo, Shanqing Li(D, Heng Zhao (i) and Zhihong Zhang (1)

Correction to: Nature Biomedical Engineering https:/doi.org/10.1038/s41551-021-00746-5, published online 15 June 2021.

In the version of this Article initially published, there was an error in the formulation of the Competing interests statement and an omission in the Acknowledgements section.

Specifically, in the Competing interests statement, the text "X.G. is a consultant for Burning Rock Biotech" has been removed. Xin Guo was not operating in a personal consultant capacity for Burning Rock Biotech for the work presented in this Article. Her contributions were strictly to the scientific endeavor, with no overlap with Burning Rock Biotech. However, Burning Rock Biotech has provided general gift funding to the University of California at Berkeley, Guo's institution, that is not attached to any particular research project. Thus, the Acknowledgements section has been amended to include the following text: "We acknowledge research funding, not attached to this study or to any research project or collaboration, from Burning Rock Biotech to the University of California at Berkeley."

The changes have been made to the online version the article.

Published online: 30 September 2021

https://oi.org/10.1038/s41551-021-00818-6

(๑) The Author(s), under exclusive licence to Springer Nature Limited 2021 